\title{
O conhecimento popular acerca de plantas medicinais: um estudo com famílias
}

\section{rurais}

\author{
Popular knowledge about medicinal plants: a study with rural families \\ El conocimiento popular acerca de plantas medicinales: un estudio con familias rurales
}

Recebido: 08/12/2021 | Revisado: 13/12/2021 | Aceito: 19/12/2021 | Publicado: 20/12/2021

Gabriele Schek

ORCID: https://orcid.org/0000-0001-8476-788X Faculdades Integradas Machado de Assis, Brasil E-mail: gabriele@ fema.com.br

Paulo Roberto Mix

ORCID: https://orcid.org/0000-0002-5762-7678 Faculdades Integradas Machado de Assis, Brasil

E-mail.paulomix@fema.com.br

Daniele Rita Assmann Kochhann

ORCID: https://orcid.org/0000-0002-6764-3823 Faculdades Integradas Machado de Assis, Brasil E-mail: danielekochhann@gmail.com

Daiana Gomes

ORCID: https://orcid.org/0000-0002-6764-3823 Faculdades Integradas Machado de Assis, Brasil E-mail: daiagomesd@hotmail.com

Larissa Rigon

ORCID: https://orcid.org/0000-0002-7595-6280 Faculdades Integradas Machado de Assis, Brasil E-mail: larissa.rigon@outlook.com

Andressa da Ronch

ORCID: https://orcid.org/0000-0003-1336-2740 Faculdades Integradas Machado de Assis, Brasil E-mail: daronchandressa@gmail.com

\begin{abstract}
Resumo
O uso de plantas medicinais como recurso terapêutico compõe uma atividade desenvolvida desde as mais antigas civilizações e atualmente representa uma das principais formas de autocuidado à saúde da população. O objetivo deste estudo é avaliar o conhecimento das famílias rurais acerca da utilização de plantas no cuidado em saúde. Trata-se de uma pesquisa qualitativa, exploratória e descritiva realizada com 20 famílias que moram na zona do rural de um município localizado na região noroeste do Estado do Rio Grande do Sul. A coleta de dados ocorreu por meio de questões semiestruturadas e para análise de dados utilizou-se a análise temática. O projeto obteve aprovação do Comitê de Ética em Pesquisa, sob $\mathrm{n}^{\circ}$ de parecer 2.628.655. Os resultados apontam que as famílias entrevistadas utilizam plantas medicinais principalmente para sintomas digestivos e respiratórios, alívio de inflamação e como ansiolíticos. O conhecimento referente a estas espécies provém de gerações antepassadas, mas também de vizinhos e da comunidade. Neste contexto, conclui-se a importância do conhecimento dos profissionais de saúde a respeito da utilização de plantas medicinais, tendo em vista que estes podem tornarem-se mediadores entre o saber popular e o conhecimento científico. Palavras-chave: Plantas medicinais; Conhecimento; Família.
\end{abstract}

\begin{abstract}
Plants with medical properties have been used as a therapeutic resource since ancient ages, and this practice currently represents one of the main interventions inself-care in the population. The goal of this study is to assess the knowledge of rural families about the use of medicinal plants in health care. It consists of a qualitative, exploratory and descriptive study performed with twenty families living in the rural area of a city located in the Northwest region of the Rio Grande do Sul State. Data collection was performed through semi-structured questions, and data analysis was carried out through thematic analysis. The project was approved by a Research Ethics Committee under number 2.628.655. Results reveal that the participants use medicinal plants mostly for digestive and respiratory symptoms, for relief of inflammation, and as anxiolytics. Their knowledge concerning plant species comes mainly from previous generations, but also from neighbors and the community. In this context, it is important that healthcare professionals know about medicinal plants and their use in the community, as they can become mediators between popular and scientific knowledge.
\end{abstract}

Keywords: Medicinal plants; Knowledge; Family. 


\begin{abstract}
Resumen
El uso de plantas medicinales como recurso terapéutico es una actividad desarrollada desde las civilizaciones más antiguas y representa en la actualidad una de las principales formas de autocuidado para la salud de la población. El objetivo de este estudio es evaluar el conocimiento de las familias rurales sobre el uso de plantas en el cuidado de la salud. Se trata de una investigación cualitativa, exploratoria y descriptiva realizada con 20 familias que viven en el área rural de un municipio ubicado en la región noroeste del Estado de Rio Grande do Sul. La recolección de datos se realizó a través de preguntas semiestructuradas y para análisis de datos, se utilizó análisis temático. El proyecto fue aprobado por el Comité de Ética en Investigación, bajo dictamen número 2.628.655. Los resultados muestran que las familias entrevistadas utilizan plantas medicinales principalmente para síntomas digestivos y respiratorios, alivio de la inflamación y como ansiolíticos. El conocimiento sobre estas especies proviene de generaciones anteriores, pero también de los vecinos y la comunidad. En este contexto, se concluye la importancia del conocimiento de los profesionales de la salud sobre el uso de plantas medicinales, considerando que pueden convertirse en mediadores entre el conocimiento popular y el conocimiento científico.
\end{abstract}

Palabras clave: Plantas medicinales; Conocimiento; Familia.

\title{
1. Introdução
}

O uso de plantas medicinais como recurso terapêutico compõe uma atividade desenvolvida desde as mais antigas civilizações (Guimarães, et al., 2021). O homem pré-histórico já empregava e sabia diferenciar as plantas comestíveis daquelas que podiam ajudar na cura de algumas enfermidades, para tanto, valiam-se de suas próprias experiências empíricas de acerto e erro e da observação do uso de plantas pelos animais. (Rocha, et al., 2015).

O conhecimento popular é um dos principais responsáveis pelos saberes e práticas adotadas envolvendo a utilização de plantas medicinais e o cuidado em saúde. Este conhecimento vem contribuindo de forma relevante para a divulgação das potencialidades terapêuticas das espécies, despertando interesse de pesquisadores e profissionais de saúde (Zeni, et al., 2017).

Apesar do significativo avanço tecnológico e das melhorias apresentadas no âmbito das políticas públicas e serviços de saúde, o uso de plantas medicinais mostra-se ainda é bastante freqüente. De acordo com estimativas da Organização Mundial de Saúde, aproximadamente $80 \%$ da população mundial utiliza e confia em produtos medicinais de origem vegetal. (WHO, 2002).

Na perspectiva das famílias que vivem no ambiente rural, estudos apontam para a ampla utilização de plantas medicinais para o cuidado em saúde. Neste contexto, a autonomia das comunidades rurais se intensifica à medida que se encontram distantes dos centros urbanos, compartilhando assim de uma realidade subjetiva, o que fortalece os vínculos locais. (Lima et al., 2014).

Pesquisas revelam que a crescente utilização de recursos naturais para o cuidado em saúde pode ser influenciada pelos custos relacionados a aquisição de fármacos industrializados. É fundamental considerar também que, grande parte da população brasileira ainda não possui acesso igualitário aos serviços do Sistema Único de Saúde, sendo assim, as plantas medicinais e sua inserção no SUS podem contribuir para democratizar o acesso á saúde por parte da população (Silva \& Barros, 2021; Rocha, et al., 2021).

Ademais, o aumento da utilização de plantas medicinais também pode estar associado ao sentimento de que a medicina e os tratamentos convencionais não têm conseguido atender o indivíduo em sua integralidade, em consequiência, as plantas medicinais ganham protagonismo no contexto onde são utilizadas. Há um processo mais ativo dos indivíduos no que se refere ao processo saúde/doença, contribuindo para que estes busquem outras formas de exercer o cuidado em saúde (Rocha, et al. 2015; Schek, et al., 2015; Botelho, et al., 2014).

Todavia, é preciso atentar para os problemas relacionados ao consumo de plantas medicinais dentre eles, a falta de orientação por parte dos usuários, que geralmente se automedica e a carência de conhecimento por parte dos profissionais de saúde que não possuem clareza suficiente sobre as concepções corretas de uso de plantas medicinais o que justifica a realização de estudos abordando esta temática.

Reconhecer o saber popular referente a utilização de plantas medicinais requer dos profissionais de saúde não apenas o registro do conhecimento tradicional, mas o aprofundamento acerca dos requisitos de segurança e eficácia que envolvem a 
utilização desta prática no cuidado à saúde. Diante do exposto, o objetivo deste estudo é identificar o conhecimento das famílias rurais acerca da utilização de plantas no cuidado em saúde

\section{Metodologia}

Estudo exploratório, descritivo, de natureza qualitativa, integrado a um macro-projeto intitulado: A utilização de plantas medicinais e o cuidado em saúde em famílias rurais de um município da região Noroeste do Rio Grande do Sul, desenvolvido pelo Curso de Bacharelado em Enfermagem das Faculdades Integradas Machado de Assis. Para Minayo (2014), a pesquisa qualitativa responde a questões particulares. Ela se ocupa com um nível de realidade que não pode ser quantificado, trabalhando com uma gama de significados, motivos, aspirações, crenças, valores e atitudes. A pesquisa exploratória possibilita ao pesquisador acrescentar sua experiência em torno do problema a ser estudado e os estudos descritivos têm como objetivo conhecer a comunidade, suas características, valores, pessoas e seus problemas (Triviños, 2008).

O estudo foi desenvolvido com 20 famílias que moram na zona do rural de um município de pequeno porte localizado na região noroeste do Estado do Rio Grande do Sul e que atenderam aos seguintes critérios de inclusão: ter mais de 18 anos, morar na zona rural a pelo menos 5 anos e ter conhecimento acerca da utilização de plantas medicinais para o cuidado em saúde.

As famílias foram recrutadas com auxílio de Agentes Comunitários de Saúde que atuam em 4 Unidades de Estratégia de Saúde da Família localizadas no interior do município destinadas ao atendimento da população rural, desencadeando assim a cadeia de informantes, conforme a metodologia Snowball (Hancock \& Gil, 2011). Os dados foram coletados entre os meses de outubro de 2018 e maio de 2019, por meio de entrevistas semiestruturadas realizadas no domicílio destas famílias, com data e hora previamente agendadas, com duração média de 45 minutos.

As entrevistas foram gravadas com o propósito de não ocorrer perdas nas informações coletadas, sempre com o consentimento dos informantes. Cada uma das famílias elegeu um de seus membros para participar da pesquisa, com base, segundo os informantes, no conhecimento prévio sobre práticas de cuidado adotadas no contexto familiar. Para preservar o anonimato, cada participante foi identificado pela letra $\mathrm{F}$ seguida do número que corresponde à ordem em que foram entrevistadas. Exemplo: (F1, F2,...). Para análise de dados utilizou-se a análise temática.

O estudo foi aprovado pelo Comitê de Ética em Pesquisa da Universidade Regional Integrada do Alto Uruguai e Missões em 30 de abril de 2018 sob o número de parecer 2.628.655 e CAAE $n^{\circ}$ 86710518.0.0000.5354. Em todas as etapas do estudo foram respeitadas a Resolução nº 466/12 (Brasil, 2012).

\section{Resultados}

Dos 20 participantes da pesquisa, 17 eram do sexo feminino e três do sexo masculino, com idade entre 40 e 86 anos. A maioria dos entrevistados possui descendência alemã e italiana e a religião predominante é a religião católica. Com relação ao grau de escolaridade, 15 dos entrevistados possuem primeiro grau completo e cinco deles possuem segundo grau completo.

Em análise, observou-se que o conhecimento acerca da utilização das plantas medicinais foi herdado das gerações mais antigas, sendo aprimorado por meio de livros e de materiais disponíveis na internet.

Este conhecimento vem de experiências antigas, isso a gente trás de berço. Minha avó já utilizava, passou o que sabia para a minha mãe e foi assim que a gente aprendeu sobre as plantas. (F1)

Outro fator evidenciado é que, em situações de dúvida referentes à ação terapêutica, a dosagem e a identificação de uma planta medicinal, a maioria das famílias solicita apoio aos vizinhos mais próximos para obter tais informações. 
Geralmente a gente comenta com alguma vizinha, uma vai perguntando para a outra para que serve este ou aquele chá. (F13)

Nós temos um grupo de mulheres que se reúne. Nós fazemos Olina caseira que vai 23 ou 26 folhas de chás. Neste momento a gente aproveita a troca um pouco de conhecimento, trazendo coisas novas em relação às plantas. (F1)

É preciso destacar que os profissionais de saúde não se constituem como fonte de informação no que tange a utilização de plantas medicinais. Segundo o relato dos entrevistados muitos profissionais de saúde não expressam interesse sobre formas alternativas de cuidado, outros parecem não possuir conhecimento sobre plantas medicinais mostrando-se indiferentes quanto sua utilização.

Uma vez eu perguntei para o médico, mas ele me disse que se eu achar que está ajudando eu posso tomar. Eles não interferem muito, nem positivo nem negativamente. (F12)

A médica me disse uma vez que não adiantava eu tomar chá, que no meu caso só com medicação. (F7)

As famílias entrevistadas habitam na zona rural, tendo a terra como a principal fonte de renda. Nas propriedades, observa-se o plantio de soja, trigo e milho, além da agricultura famílias. Tais atividades são realizadas essencialmente realizadas com mão de obra familiar o que leva o envolvimento de todos com a terra. Neste contexto, os recursos naturais são muito valorizados, assim, as 20 famílias entrevistadas deste estudo citaram 96 espécies de plantas medicinais com indicações terapêuticas distintas.

A utilização de plantas medicinais é mais freqüente frente a problemas digestivos e respiratórios. Para os primeiros problemas citados, as famílias citam através do nome popular plantas como Losna, Marcela e Espinheira Santa Já para os problemas relacionados às vias respiratórias, as famílias destacam o Guaco e folha de Laranjeira

Questionados sobre os cuidados com a utilização destas plantas, algumas famílias destacam apenas os cuidados relacionados ao consumo da Losna, cujo chá não deve ser consumido quente e nem em excesso pelo risco de intoxicação. Para as demais plantas citadas, as famílias não expressaram preocupação quando a dosagem e a periodicidade no consumo.

Além da utilização de plantas medicinais para os problemas de saúde supracitados, no conhecimento das famílias entrevistadas, algumas espécies possuem efeito anti-inflamatório, dentre elas estão a Marcela, Graviola e a Tansagem. As famílias reconhecem estas espécies através do nome popular e afirmam que as utilizam amplamente frente a dores de garganta além de utilizá-las para lavar ferimentos e lesões de pele.

Destacam-se ainda as plantas que, no conhecimento das famílias, possuem efeito "calmante", dentre elas estão: Melissa, Capim-Cidreira, Poejo, Hortelã, Alecrim e folha de Maracujá, todas citadas através do seu nome popular. Nesta categoria, as plantas medicinais são indicadas para crianças, pois além de calmante estas espécies também contribuem para problemas como cólicas frequentemente observados em recém-nascidos e crianças pré-escolares. Nesta categoria as famílias relatam alguns cuidados principalmente com relação à dosagem ofertada a criança, ou seja, os chás não devem substituir a ingestão de água e devem ser utilizados somente em situações pontuais.

O conhecimento relacionado à utilização de plantas medicinais ainda envolve a produção de produtos derivados de distintas espécies. 
Meu filho estava com os triglicerídeos elevados. Eu fiz uma infusão de salsa parrilha, alcachofra, quebra pedra e mais um chá que não recordo o nome ....Infusão na cachaça. Ele tomou 30 gotas 3 vezes ao dia. Ele tomou 18 dias, repetiu o exame e tinha baixado muito. (F1)

Durante o verão eu pego uns 20 chás e faço um xarope para a tosse. Faço para o ano todo. Os filhos e os netos vêm e já dou para eles levarem. (F20)

Todas as plantas medicinais utilizadas pelas famílias rurais deste estudo foram citadas através do seu nome popular, indicando que as famílias não possuem familiaridade e/ou conhecimento com relação à identificação cientifica das espécies que fazem uso.

\section{Discussão}

As famílias rurais entrevistadas possuem conhecimento sobre plantas medicinais e estes provem majoritariamente de seus antepassados. Para alguns autores este conhecimento está fundamentado em crenças, valores e na cultura que é estabelecida no âmbito de cada família constituindo-se como um importante campo gerador de conhecimento etnobotânico. (Lima, et al., 2014). Este conhecimento permite averiguar a relação entre o homem e as plantas medicinais podendo refletir em descobertas de novos compostos farmacológicos e indicações terapêuticas. (Cajaiba, et al., 2019).

Além do conhecimento familiar acerca da utilização de plantas medicinais, muitos estudos demonstram que estes saberes também são adquiridos junto a múltiplas fontes, especialmente entre pessoas da comunidade, participação em pastorais e leituras de livros. (Lima, et al., 2019; Lopes, 2016). Todavia, atenta-se pelo fato da pouca participação dos profissionais de saúde no que tange ao conhecimento e as orientações dadas às famílias sobre a utilização de plantas medicinais.

Autores discutem que muitos profissionais mostram-se resistentes a modelos de atenção à saúde que não priorizem o modelo biomédico. Esta atitude pode estar respaldada pela falta de conhecimento cientifico sobre a utilização de recursos naturais como alternativa segura e eficaz, refletindo muitas vezes em conflitos culturais e científicos. (Rocha, et al., 2015). Estudo de revisão sobre a temática plantas medicinais apontou a carência de discussões e esclarecimentos sobre o assunto durante os cursos de graduação da área da saúde, gerando lacunas de conhecimento principalmente associada a assistência daquelas populações que tem por costume a tradição a utilização de plantas medicinais (Nunes \& Maciel, 2016).

Nesta perspectiva, torna-se fundamental que as equipes de saúde ao planejarem a assistência à família ou comunidade passem a considerar a cultura dos indivíduos e seus recursos disponíveis, dentre eles as plantas medicinais. Para tanto, há a necessidade de conhecimento das propriedades terapêuticas das plantas a serem usadas, do preparo, da indicação, dos cuidados e da dosagem.

Com intuito de valorizar o conhecimento popular e fortalecer a atuação dos profissionais de saúde com relação a temática plantas medicinais, o Ministério da Saúde através do decreto pelo ${ }^{\circ}$ 5.813/2006 criou a Política Nacional de Plantas Medicinais e Fitoterápicos com vistas a garantir à população brasileira o acesso seguro e o uso racional de plantas medicinais e fitoterápicos, promovendo o uso sustentável da biodiversidade, o desenvolvimento da cadeia produtiva e da indústria nacional. (Brasil, 2006). Adicionalmente, o Ministério da Saúde elaborou a Relação Nacional de Plantas Medicinais de interesse ao SUS (RENISUS) listando 71 espécies vegetais. (Brasil, 2009).

Ao comparar as indicações terapêuticas citadas pelas famílias com as indicações encontradas na literatura. observamse algumas convergências. À exemplo, cita-se a Marcela (Achyrocline satureioides) e a Espinheira Santa (Maytenus ilicifolia) ambas com efeitos positivos frente a problemas digestivos e distúrbios gástricos. (Brasil, 2009; Lorenzi \& Matos, 2002; Schek, et al., 2015; Almeida, et al., 2015). Com relação ao Guaco (Mikania sp) e a indicação terapêutica atribuída pelas famílias rurais 
esta vai ao encontro da Resolução RDC número 10 da ANVISA, que aconselha a utilização desta espécie para casos de gripes, bronquites e como expectorante (Brasil, 2010). Já a folha de laranjeira (Citrus sinensis (L.) Osbeck) é indicada para problemas respiratórios, corroborando com o que foi relatado pelos sujeitos desta pesquisa. (Piriz, 2013).

Com relação às plantas medicinais com efeitos antiinflamatórios a Tansagem e Marcela possuem seus efeitos antiinflamatórios reconhecidos em estudo publicado (Marmitt, et al., 2015).

Das plantas medicinais citadas neste estudo como calmantes, 2 delas convergem com o que a literatura cientifica: a melissa (Melissa officinalis), capim-cidreira (Cymbopogon citratus) possuem indicação para casos leves de ansiedade. Já o poejo (Mentha pulegium) e o Hortelã (Mentha $s p$ ) possuem indicações cientificas divergentes das famílias rurais, visto que o poejo pode ser utilizado contra afecções respiratórias e como expectorante e a hortelã possui efeito positivo contra problemas digestivos, amenorréia, resfriados e ajuda a aumentar a micção. (Lorenzi \& Matos; 2002).

No contexto das práticas de cuidado envolvendo a utilização de plantas medicinais, é preciso atentar pelos riscos do seu consumo exagerado. Em extensa revisão bibliográfica identificou-se o Guaco (Mikania sp.) interage com alguns antibióticos como tetraciclinas, cloranfenicol, gentamicina, vancomicina e penicilina e a Hortelã (Mentha sp): pode provocar a inibição da absorção de ferro pelas proteínas sangüíneas, além de aumentar os níveis sanguíneos de drogas como a sinvastatina. (Lorenzi \& Matos; 2002). Diante do exposto, é fundamental avaliar os riscos de intoxicação causados pelo uso indevido de algumas espécies. A não observância às doses e o cuidado na identificação precisa da espécie pode acarretar prejuízos à saúde.

Assim, é importante que profissionais de saúde busquem se instrumentalizar com vistas a compreender as distintas ações de cuidado realizadas pelas famílias rurais, especialmente a utilização de plantas medicinais. O conhecimento acerca desta prática permite aos profissionais de saúde tornarem-se mediadores entre o saber popular e o conhecimento científico, construindo novos saberes em modos de agir (Oliveira, et al., 2020).

\section{Considerações Finais}

Este estudo evidenciou que a utilização de plantas medicinais entre as famílias rurais é uma prática frenquentemente adotada frente a determinados problemas de saúde. O conhecimento referente às plantas que podem ser utilizadas geralmente foi herdado dos antepassados, todavia quanto há dúvidas em relação a identificação de alguma espécie os vizinhos e a comunidade passam a se tornar a principal fonte de informação.

Além disso é preciso destacar o pouco envolvimento dos profissionais de saúde em relação aos cuidados ao consumo de chás, atribuído pela falta de conhecimento ou, por não considerarem os efeitos terapêuticos atribuídos a algumas espécies vegetais.

As plantas medicinais utilizadas pelas famílias rurais são reconhecidas pelo seu nome popular e por isso as famílias entrevistadas citaram-nas dessa forma. Isso se constitui em uma limitação deste estudo, visto que para uma única espécie vegetal, vários nomes populares podem ser conferidos. Ao comparar as indicações terapêuticas atribuídas pelas famílias rurais com estudos referentes a temática, observaram-se convergências para algumas espécies o que demonstra o conhecimento das famílias entrevistadas. Todavia, não manifestação referente aos cuidados no que tange o consumo exagerado das plantas medicinais citadas, o que diverge com a literatura cientifica que aponta alguns cuidados a serem adotados com algumas espécies.

Com base no que foi discutido, este estudo aponta para a necessidade de mudanças na relação famílias/ profissionais com relação as práticas de saúde que envolvam a utilização de plantas medicinais visto os riscos associados, que podem se expressar pela falta de identificação correta de uma espécie e pelo seu consumo exagerado. Propõe-se aos profissionais de saúde uma aproximação com o conhecimento popular acerca das plantas medicinais, devido ao protagonismo que estas assumem como práticas de cuidado no contexto das famílias rurais. 


\section{Referências}

Almeida, C., Barbieri, R. L., Ribeiro, M. V., Lopes, C. V \& Heck, R. M. (2015). Espinheira-santa (Maytenus ilicifolia Mart. ex Reiss.): saber de erveiros e feirantes em Pelotas (RS). Revista Brasileira Plantas Medicinais, 17(4):722-729. 10.1590/1983-084X/14_003

Botelho, J. M., Lamano-Ferreira, A. P. N., \& Ferreira, M. L. (2014). Prática de cultivo e uso de plantas domésticas em diferentes cidades brasileiras. Revista Ciência Rural.44(10): 1810-15. 10.1590/0103-8478cr20131036

BrasiL. (2010). ANVISA. Resolução RDC $n^{\circ} 10$, de 9 de março de 2010. Dispõe sobre a notificação de drogas vegetais junto à Agência Nacional Sanitária (ANVISA) e dá outras providências. https://bvsms.saude.gov.br/bvs/saudelegis/anvisa/2010/res0010_09_03_2010.html

BrasiL. (2006). Ministério da Saúde. Secretaria de ciência, tecnologia e insumos estratégicos. Política nacional de plantas medicinais e fitoterápicos. Ministério da Saúde. https://bvsms.saude.gov.br/bvs/publicacoes/politica_nacional_fitoterapicos.pdf

Brasil. (2009). Ministério da Saúde. RENISUS - Relação Nacional de Plantas Medicinais de interesse ao SUS. https://bvsms.saude.gov.br/bvs/sus/pdf/marco/ms_relacao_plantas_medicinais_sus_0603.pdf

Brasil. (2012). Ministério da Saúde. Conselho Nacional de Saúde. Resolução n. 466, de 12 de dezembro de 2012. Aprova diretrizes e normas regulamentadoras de pesquisas envolvendo seres humanos. https://conselho.saude.gov.br/resolucoes/2012/Reso466.pdf

Cajaiba, R.L., Silva, W. B., Sousa, R. D. N., \& Sousa, A. S. (2019). Levantamento etnobotânico de plantas medicinais comercializadas no município de Uruará, Pará, Brasil. Revista Biotemas. 29 (1): 115-131. 10.5007/2175-7925.2016v29n1p115

Guimarães, B. M., Ramos, K. A., Souza, M. C., Franco, M. L., Alves, C. C. S., Carli, A. P., \& Coqueiro, J. M. (2021). Práticas terapêuticas com plantas medicinais para o tratamento do Diabetes Mellitus. Research, Society and Development, 10(10): e474101018874. 10.33448/rsd-v10i10.18874.

Handcock, M. S., \& GILE, K. J. (2011). On the Concept of Snowball Sampling. Sociological Methodology. 41(1): 367-371. 10.1111/j.1467-9531.2011.01243.x

Lima, A. R. A., Heck, R. M., Vasconcelos, M. K. P., \& Barbieri RL. (2014). Ações de mulheres agricultoras no cuidado familiar: uso de plantas medicinais no sul do Brasil. Texto Contexto Enfermagem. 23(2): 365-72. 10.1590/0104-07072014004080012

Lopes, C. V. (2016). O cuidado no sistema informal de saúde: um enfoque cultural no urbano e rural em Pelotas/RS. [Tese, Programa de Pós-Graduação em Enfermagem. Universidade Federal de Pelotas]. https://docs.bvsalud.org/biblioref/2018/02/879298/tesededoutoradocarolinelopes061016.pdf

Lorenzi, H \& Matos, F.J. A. (2002). Plantas Medicinais no Brasil: nativas e exóticas. Plantarum.

Marmitt, D. J., Rempel, C., Goettert, M., \& Silva, A. C. (2015). Medicinal Plants RENISUS With Potential Anti-inflammatory: Systematic Review In Three Scientific Databases. Revista Fitos, 9(2): 73-159. 10.5935/2446-4775.20150011129.

Minayo, M. C. S. (2014). O Desafio do Conhecimento: pesquisa qualitativa em saúde. (6a ed.), Hucitec.

Nunes, J., \& Maciel, M. (2016). A importância da informação do profissional de enfermagem sobre o cuidado no uso das plantas medicinais: uma revisão de literatura. Revista Fitos, 10(4): 375-547. 10.5935/2446-4775.20160037

Oliveira, K. K. B., Rocha, G. M. M., Brito, M. G. A., Silva, M. A., \& Oliveira, G. A. L (2020). Medicinal plants used to treat gastrointestinal disorders: an integrative review. Research, Society and Development, 9(9), p. e438997164, 2020. 10.33448/rsd-v9i9.7164.

Piriz , M. A., Mesquita, M. K., Cavada, C. T., Palma, J. P., Ceolin, T., \& Heck, R. M. (2013). Uso de plantas medicinais: impactos e perspectivas no cuidado de enfermagem em uma comunidade rural. Revista Eletrônica de Enfermagem [Internet], 15(4):992-9. 10.5216/ree.v15i4.19773

Rocha, F. A. G., Araújo, M. F. F., Costa, N. D. L., \& Silva, R. P. (2015). O uso terapêutico da flora na história mundial. Holos, 31 (1) 49-61. 10.15628/holos.2015.2492

Rocha, L. P. N., Alves, J. V. O., Aguiar, I. F. S., Silva, F. H., Silva, R. L., Arruda, L. G., Filho, B. V. D. R., Amorim, L. C., Silva, P. M., \& Silva, M. V. (2021). Use of medicinal plants: History and relevance, Research, Society and Development, 10 (1), p. e44101018282. 10.33448/rsd-v10i10.18282.

Schek, G., Barbieri, R. L., \& Heck, R. M. (2015). Processo saúde/doença e cuidado em famílias descendentes de pomeranos: contribuições para a enfermagem. Revista de Enfermagem [on-line],11 (11): 54-62. http://periodicos.fw.uri.br/index.php/revistadeenfermagem/article/view/1626/1972

Schek, G., Barbieri., R. L., Heck, R. M \& Marchi, M. M. (2015). Plantas medicinais no cuidado à saúde em famílias descendentes de pomeranos no sul do Brasil. Enfermeria Comunitaria. 11(1). http://www.index-f.com/comunitaria/v11n1/ec9736r.php

Silva, A. F. L., \& Barros, L. A. S. (2021). Avaliação das práticas de uso de plantas medicinais no Município de Caxias-MA. Research, Society and Development, 10(4) e10010413832. 10.33448/rsd-v10i4.13832

Triviños, A. N. S. (2008). Introdução à pesquisa em ciências sociais: a pesquisa qualitativa em educação. Atlas.

Zeni, A. L. B., Parisotto, A. V., Mattos, G., \& Helena, E. T. S. (2017). Utilização de plantas medicinais como remédio caseiro na Atenção Primária em Blumenau, Santa Catarina, Brasil. Ciência Saúde Coletiva, 22(8):2703-12. 10.1590/1413-81232017228.18892015

World Health Organization. (2002). Policy Pespectives on Medicines. Traditional Medicine - Growing Needs and Potencial. World Health Organization, 2002. 